\title{
High throughput fabrication of cell spheroids by templating water-in-water Pickering emulsions
}

Received 00th January 20xx, Accepted 00th January 20xx

DOI: $10.1039 / \times 0 \times x 00000 x$
Anupam A.K. Das, ${ }^{a}$ Benjamin Filby, ${ }^{a}$ Daniel Geddes, ${ }^{a}$ Deborah Legrande ${ }^{a}$ and Vesselin N. Paunov*a rsc.li/materials-horizons

We report a simple technique for the high throughput generation of tissue spheroids based on encapsulation of dispersed adherent cells in particle-stabilised water-in-water emulsions. We demonstrate the cell encapsulation and spheroid generation using fibroblast cells which can find applications in 3D culturing, tissue engineering, inks for bioprinting and cell therapy.

Recently, we have seen a great progress in 3D cell culture technologies and culture systems used for landmark developments in organoids with realistic microstructure, ${ }^{1}$ tissue engineering and simulating organ level physiology. ${ }^{2}$ The cells can form mini tissues or tissue spheroids in the form of a 3D cell culture model..$^{3-5}$ Cell spheroids has been actively used for production of 3D tumour models ${ }^{6-8}$ and tissue reconstruction or organ bioprinting. ${ }^{5,9}$ Spheroids of adherent cells can be formed using different processes for cell clustering where they adhere to each other rather than to a substrate. The current processes of cell spheroid production involve spinner culture, NASA rotary culture and non-adhesive surfaces. ${ }^{10}$ In order to control the size of individual spheroids, the hanging-drop culture ${ }^{11,12}$ and 3D culturing in microwells ${ }^{13}$ are extensively used. Alternative techniques are based on micro-rotational flow ${ }^{14}$ and on the magneto-Archimedes effect ${ }^{15}$ to form similarly sized tissue spheroids.

In the hanging drop method, small pendant drops of cell culture are hung from the underside of a culture dish lid, held in their position by surface tension forces. The cells concentrate at the bottom of the drops due to the gravity and adhere to each other, which results in the formation of the spheroids. ${ }^{16-18}$ The same method was also used to make spheroid assemblies containing different cell types. ${ }^{19,20}$ Bio-inks based on viable and similarly sized cell spheroids allow them to be bio-printed into 3D structures and organs where they can proliferate and grow more efficiently than single cels. ${ }^{21}$

\footnotetext{
a. School of Mathematics and Physical Sciences (Chemistry), University of Hull, Cottingham Road, Hull, HU67RX, UK.

*Corresponding author: V.N.Paunov@hull.ac.uk, Tel: +44 (0)1482 465660.

* Electronic Supplementary Information (ESI) available: DOI: $10.1039 / x 0 x x 00000 x$
}

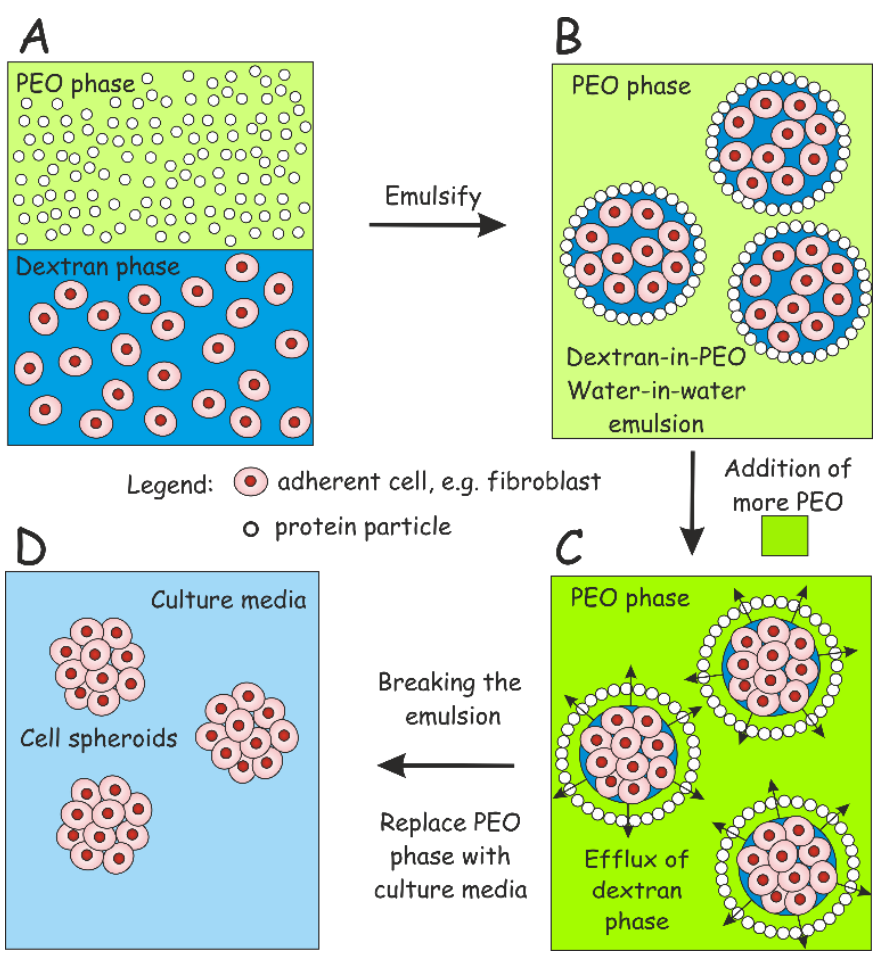

Figure 1 Schematic of the preparation of tissue spheroids by encapsulation of adherent cells in a dextran-PEO water-in-water emulsion template stabilised by protein particles. Upon emulsification, cells prefer the discontinuous dextran phase which allow their encapsulation. Adding more concentrated PEO phase causes osmotic shrinking of the cell-rich dextran drops, whose interfacial tension packs the cells into tissue spheroids. The latter are isolated by breaking the emulsion by dilution with culture media.

However, most of the current processes for generation of cell spheroids cannot be used for tissue engineering on a large scale. A new cheap and rapid technology is needed, capable of high throughput spheroid production. The current methods of production of cellosomes ${ }^{22,23}$ can serve as a possible way for larger scale production of desired spheroids. Encapsulation of cells in such structures has also been achieved by using natural biopolymers like alginate, pectin, agarose and chitosan. ${ }^{24}$ 

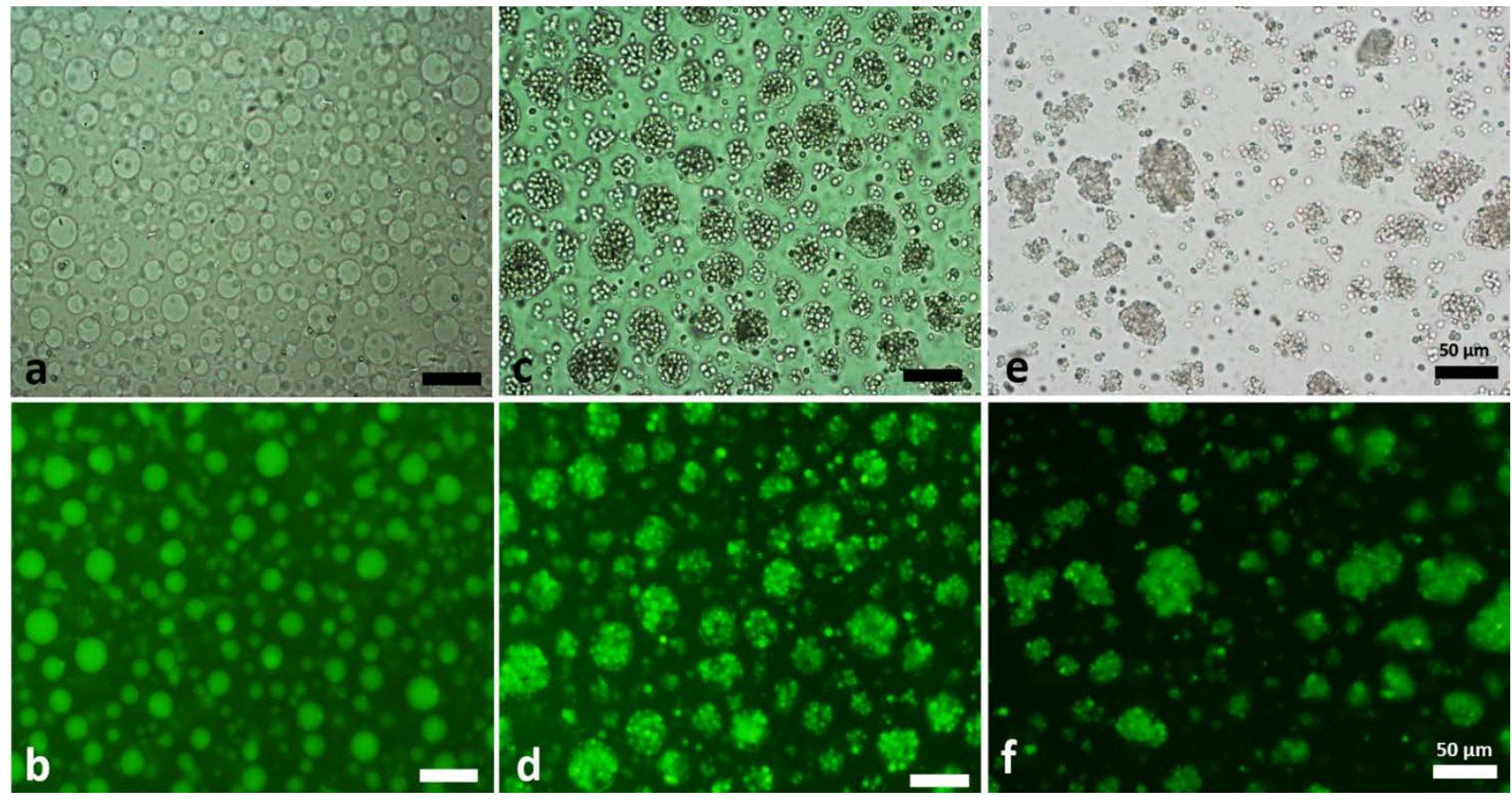

Figure 2 Optical and fluorescence microscope images of (a), (b) FITC labelled DEX droplets in PEO continuous phase stabilised using protein particles ( $\beta$-lactoglobulin) and (c), (d) FDA stained yeast cells encapsulated in the same emulsion system. (e) and (f) show a similar system with higher cell concentration in the DEX drops. All scale bars are $50 \mu \mathrm{m}$ in size.

Here we report for the first time a rapid and scalable production technique of tissue spheroids by trapping cells in droplets of water-in water Pickering emulsion stabilised by protein particles. The emulsion is formed of an aqueous solution of dextran (DEX) dispersed in a continuous phase of aqueous solution of polyethylene oxide (PEO). The schematic of our cell spheroids fabrication method is shown in Figure 1 for adherent cells which are dispersed in the DEX phase. The formation of a stable DEX/PEO emulsion leads to the cells encapsulation in the DEX droplets. Further addition of more concentrated PEO phase leads to osmotic shrinking of the DEX drops and compression of the adherent cells in spheroids. Finally, the latter are isolated by breaking the DEX/PEO emulsion by dilution with culture media. This cheap and robust technique is easy to upscale and allows rapid preparation of similarly sized cell spheroids.

Water-in-water emulsions have been stabilised effectively by the addition of solid particles. ${ }^{25,29}$ Nguyen et al. ${ }^{25}$ used $\beta$ lactoglobulin particles ( $0.6 \mathrm{wt} \%$ ) to stabilise DEX/PEO emulsion. We used similar emulsions ${ }^{25}$ stabilised with protein particles ( $\beta$ lactoglobulin or whey protein) as templates in our method for preparation of cell spheroids. The protein particles were always introduced into the PEO phase. The emulsion drops typically consisted of a DEX phase containing 2.0-5.5 wt \% dextran (M.W. $500 \mathrm{kDa}$, Fisher Scientific, UK) stained with a $50 \mu \mathrm{l}$ solution of $1 \times 10^{-5} \mathrm{M}$ FITC dextran (M.W. $500 \mathrm{kDa}$ Sigma Aldrich, UK). The DEX phase was added to the 4 wt\% PEO (M.W. 200 kDa, Sigma Aldrich, UK) slowly over the course of one minute, while homogenising at $9000 \mathrm{rpm}$ for additional 10 minutes using an IKA T25 Ultra Turrax. The detailed protocol for making the protein particles can be found in the ESI*.
Here we demonstrate the above protocol for producing stable DEX droplets in the PEO continuous phase, as evident from Figures $2 \mathrm{a}$ and $2 \mathrm{~b}$. Further, as a proof of principle, we prepared similar DEX/PEO emulsions with added $0.5 \mathrm{wt} \%$ hydrated yeast cells dispersed in the DEX phase. We found that cells prefer to accumulate in the sugar containing (DEX) phase rather than in the PEO phase. This phenomenon enabled us to trap the cells in the DEX drops dispersed in PEO phase as shown in Figures 2c and $2 \mathrm{~d}$. The viability of the cells was monitored by fluorescein diacetate (FDA) live/dead cell assay using a BX-51 fluorescence microscope equipped with a DP70 digital camera. FDA is a nonfluorescent compound which easily diffuses through the cell membranes. ${ }^{26,27}$ The uptake and hydrolysis of the nonfluorescent FDA by intracellular esterase leads to accumulation of a fluorescein within the cells if the cell membrane is intact. ${ }^{28}$ In the present work, we used yeast to demonstrate the cell encapsulation and their selective packing in the DEX phase as the individual yeast cells are easier to distinguish in the DEX drops than fibroblasts. Figures $2 \mathrm{e}$ and $2 \mathrm{f}$ show the packing of a of higher concentration yeast cells in similar volume fraction of DEX phase. Similar result was obtained by adding more concentrated PEO phase to the pre-formed DEX/PEO emulsion which shrinks the DEX drops with the entrapped cells.

Here we also demonstrate the generation of tissue spheroids from adherent fibroblast cells. We used human embryonic kidney cells (HEK293) which were cultured in media that contains $10 \%$ Fetal bovine serum (FBS) and PenStrep. 


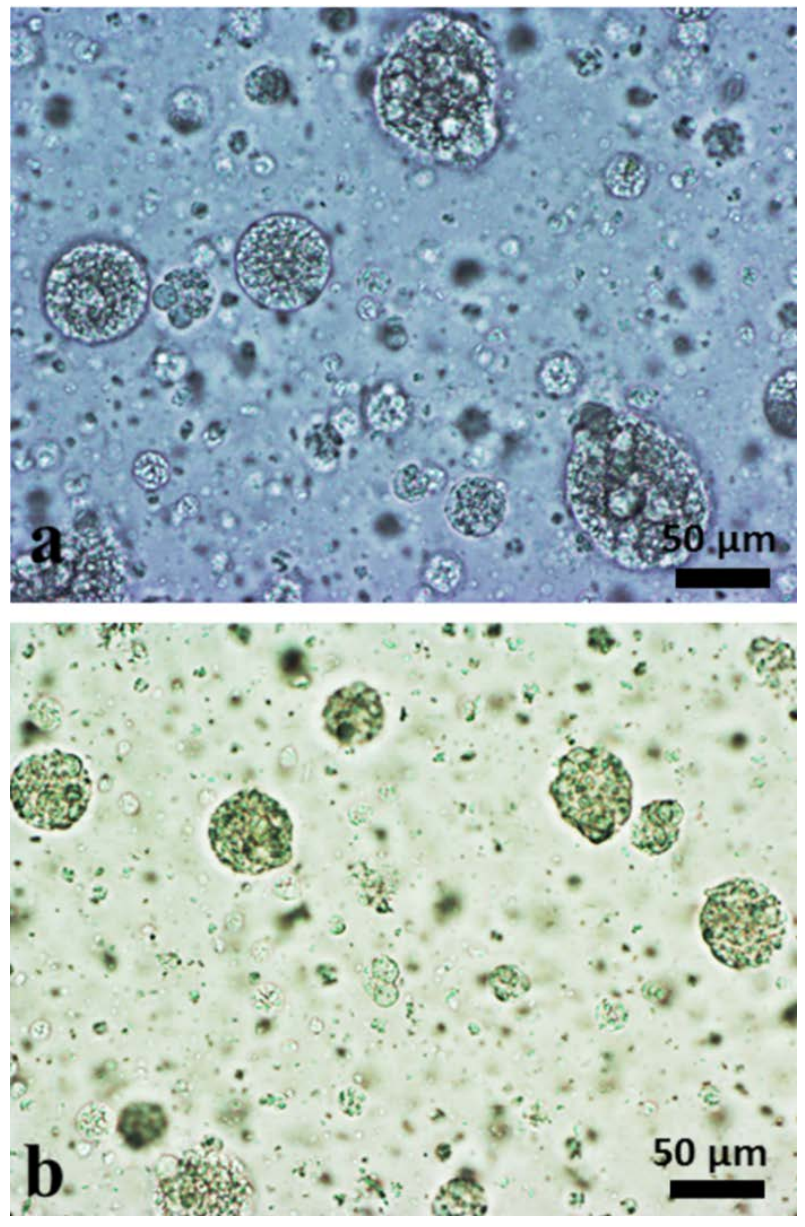

Figure 3 Optical microscopic image of (a) HEK 293 cells encapsulated in the dextran droplets in PEO continuous phase stabilised using protein particles (whey protein isolate) and (b) HEK 293 spheroids isolated from the emulsion formulation after increasing the concentration of the PEO continuous phase to $8 \%(\mathrm{~W} / \mathrm{V})$. All scale bars are $50 \mu \mathrm{m}$ in size.

The emulsion was prepared with the HEK293 cells dispersed in $5.5 \mathrm{wt} \%$ dextran aqueous phase in a continuous phase of $4 \mathrm{wt} \%$ PEO. Both the DEX and the PEO phase were made with DMEM media. The particles used for stabilising the DEX/PEO emulsion were produced using whey protein isolate (see ESI for more details) and were mixed with the PEO phase. To avoid damaging the HEK293 cells by extensive shear the homogenisation was done using a syringe with $21 \mathrm{~mm}$ gauge and $0.8 \mathrm{~mm}$ internal needle diameter) with typically 10 pumps. The same volume fractions of HEK293 cells and the original DEX phase were mixed together prior to its dispersing into the PEO phase. We found that the HEK 293 cells also preferred the DEX phase (similar to the yeast) compared to the PEO phase. Figure 3a shows the fibroblast cells successfully encapsulated in the DEX droplets. Subsequently, in order to force the fibroblast cells trapped in the DEX drops to stick together, the PEO concentration in the continuous phase was increased by several fold dilution with more concentrated PEO solution in DMEM media. This resulted in an efflux of water from the DEX phase into the PEO phase as illustrated in Figure 1. Consequently, this led to the formation of tightly packed HEK293 spheroids as evident from Figure $3 \mathrm{~b}$. This was also observed at low and high magnification microscope images HEK 293 spheroids, as shown in Figure 4a and $4 \mathrm{~b}$. The detailed protocol for the spheroid generation and the cell viability studies are described in the ESI.

The spheroids were separated by the process of gravitational sedimentation where the large spheroids sediments faster compared to single cells and the supernatant phase was extracted carefully leaving the spheroids at the bottom of the sample vials. The preservation of the cell viability in the spheroids is a critical issue during the whole emulsification and encapsulation process. We applied the FDA assay to the HEK293 cell spheroids after they have been isolated from the DEX/PEO emulsion. Figure $4 \mathrm{c}$ and $4 \mathrm{~d}$ compare the brightfield and fluorescence microscopy images of the treated HEK293 cell spheroids. One can see that the cells in the spheroids were highly active and viable which would enable them to have efficient cell growth and proliferation in various tissue engineering application. The scanning electron microscope (SEM) image in Figure $4 \mathrm{e}$ and $4 \mathrm{f}$ shows the arrangement of individual HEK293 cells in the spheroid. Fibroids cell proliferation depends crucially on the availability of neighbouring adherent cells or a solid substrate for their attachment. Generally, individual adherent cells, separated from substrate and neighbours do not proliferate as efficiently as the same cells clustered in aggregates.

In summary, we have devised a general and effective strategy for preparation of cell spheroids by encapsulating cells in the dispersed phase of a water-in-water Pickering emulsion. We demonstrate the technique with $S$. Cerevisiae (yeast) and Human embryonic kidney cells (HEK293) for the purpose of spheroid generation. Aqueous dextran solution was used as the dispersed phase in a continuous PEO aqueous phase stabilised using protein particles made from $\beta$-lactoglobulin or whey protein isolate for yeast and HEK 293 cells, respectively. The cell spheroids can produced of different sizes dependent on the cell concentration in the emulsion. The method can be further modified by maintaining a monodisperse emulsion system to produce spheroids of uniform size, although this may not be a critically needed for tissue engineering applications. It is also worth mentioning that this current method of spheroid generation is not restricted to yeast or HEK 293 cells but also can be applied to other kind of adherent cell cultures, for example tumour cells for generating suitable tumour models. This will be reported in a follow up paper. We also envisage that this method can be extended to fabricate tissue spheroids for cell therapies and bio-ink formulations for 3D organ printing. This would be made possible by the high throughput generation of cell spheroids generated using the proposed method.

\section{Conflicts of interest}

There are no conflicts of interest to declare. 

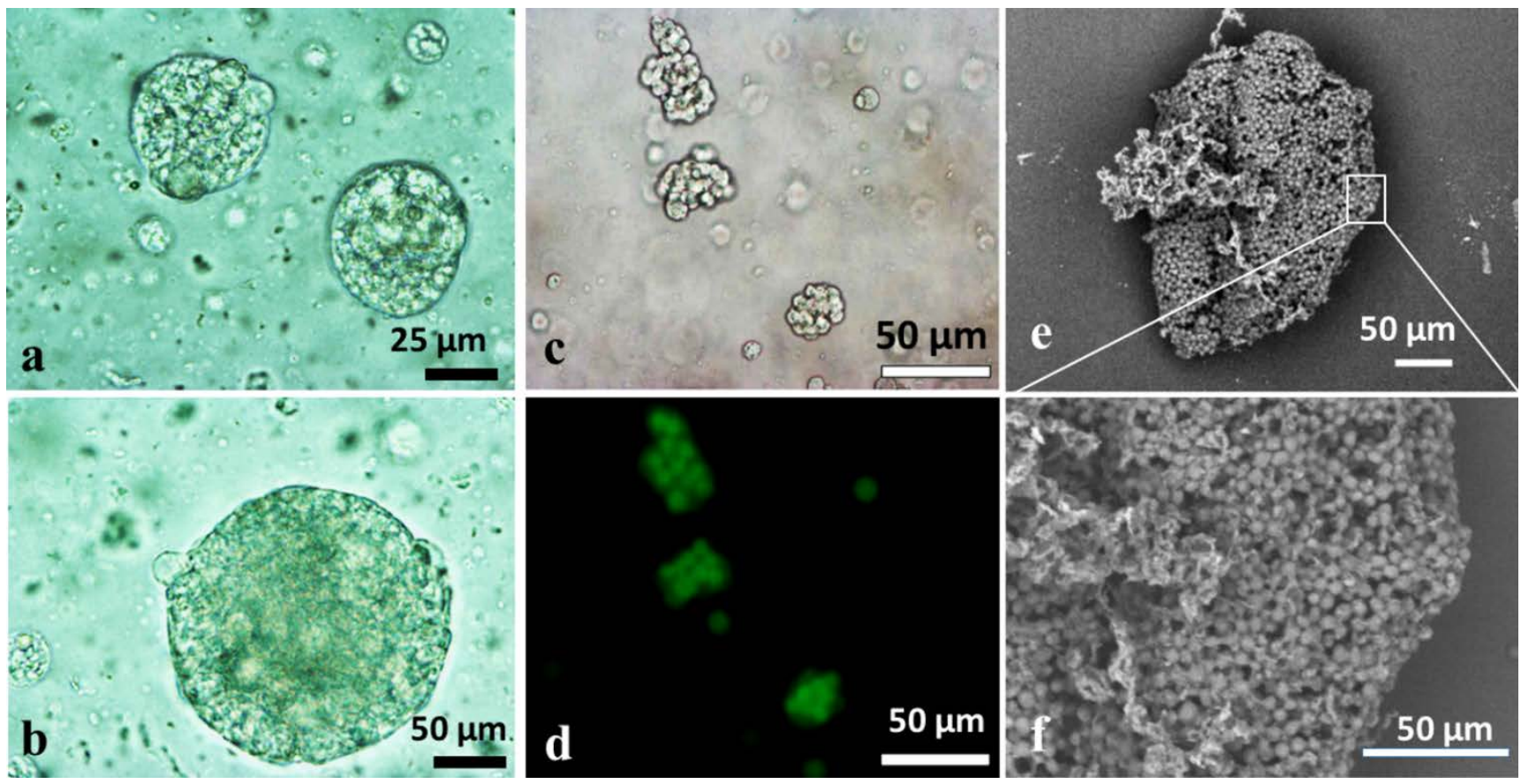

Figure 4 Optical microscopic image of (a) Low magnification HEK293 spheroids, (b) High magnification HEK293 spheroids, (c) and (d) Optical and florescence microscope images of HEK293 cell spheroids isolated from the DEX/PEO emulsion, showing the viability of the fibroblast cells by using FDA live/dead assay. (e) Scanning electron microscopic image of a HEK293 spheroid isolated from the emulsion formulation.

\section{References}

1. T. Sato, R.G. Vries, H.J. Snippert, M. Van de Wetering, N. Barker, D.E. Stange, J.H. Van Es, A. Abo , P. Kujala, P.J. Peters, H. Clevers, Nature, 2009, 459, 262.

2. D. Huh , D.C. Leslie, B.D. Matthews, J.P. Fraser, S. Jurek, G.A. Hamilton, K.S. Thorneloe, M.A. McAlexander, D.E. Ingber, Sci. Transl. Med., 2012, 4, 147.

3. K. E. Kasza, A. C. Rowat, J. Liu, T. E. Angelini, C. P. Brangwynne, G. H. Koenderink and D. A. Weitz, Current Opin. in Cell Biol., 2007, 19, 101-107.

4. A. A. K. Das, R. F. Fakhrullin and V. N. Paunov, in Cell Surface Engineering: Fabrication of Functional Nanoshells, The Royal Society of Chemistry, 2014, pp. 162-184.

5. V. Mironov, R. P. Visconti, V. Kasyanov, G. Forgacs, C. J. Drake and R. R. Markwald, Biomaterials, 2009, 30, 21642174.

6. J. Friedrich, R. Ebner and L. A. Kunz-Schughart, Internat. J. Rad. Biol., 2007, 83, 849-871.

7. Klo, M. Fischer, A. Rothermel, J. C. Simon and A. A. Robitzki, Lab on a Chip, 2008, 8, 879-884.

8. L. A. Kunz-Schughart, J. P. Freyer, F. Hofstaedter and R. Ebner, J.Biomolec.Screening, 2004, 9, 273-285.

9. R.-Z. Lin and H.-Y. Chang, Biotechn. Journal, 2008, 3, 11721184.

10. Y. Morimoto and S. Takeuchi, Biomater. Sci., 2013, 1, $257-$ 264.

11. Y. C. Tung, A. Y. Hsiao, S. G. Allen, Y. S. Torisawa, M. Ho and S. Takayama, Analyst, 2011, 136, 473-478.

12. W. G. Lee, D. Ortmann, M. J. Hancock, H. Bae and A. Khademhosseini, Tissue Eng Part C-Me, 2010, 16, 249-259.

13. M. Kato-Negishi, Y. Tsuda, H. Onoe and S. Takeuchi, Biomaterials, 2010, 31, 8939-8945.

14. H. Ota, R. Yamamoto, K. Deguchi, Y. Tanaka, Y. Kazoe, Y. Sato and N. Miki, Sensor Actuat B-Chem, 2010, 147, 359-365.

15. Y. Akiyama and K. Morishima, Appl. Phys. Lett., 2011, 98.
16. R.-Z. Lin, L.-F. Chou, C.-C. Chien and H.-Y. Chang, Cell Tissue Res., 2006, 324, 411-422.

17. J. M. Kelm, N. E. Timmins, C. J. Brown, M. Fussenegger and L. K. Nielsen, Biotechnol. Bioeng., 2003, 83, 173-180.

18. J. M. Kelm and M. Fussenegger, Trends Biotechnol., 2004, 22, 195-202.

19. T. Nicholas, D. Stefanie and N. Lars, Angiogenesis, 2004, 7, 97-103.

20. J. M. Kelm, E. Ehler, L. K. Nielsen, S. Schlatter, J. C. Perriard and M. Fussenegger, Tissue Eng, 2004, 10, 201-214.

21. V. Mironov, R. P. Visconti, V. Kasyanov, G. Forgacs, C. J. Drake and R. R. Markwald, Biomaterials, 2009, 30, 21642174.

22. R. F. Fakhrullin, M. L. Brandy, O. J. Cayre, O. D. Velev and V. N. Paunov, Phys Chem Chem Phys, 2010, 12, 11912-11922.

23. M. L. Brandy, O. J. Cayre, R. F. Fakhrullin, O. D. Velev and V. N. Paunov, Soft Matter, 2010, 6, 3494-3498.

24. L. Gasperini, J. F. Mano and R. L. Reis, J. Roy. Soc. Interface, 2014, 11, 20140817.1-19.

25. B. T. Nguyen, T. Nicolai and L. Benyahia, Langmuir, 2013, 29, 10658-10664.

26. P. Breeuwer, J. Drocourt, F. M. Rombouts and T. Abee, Applied and Environmental Microbiology, 1996, 62, 178-183.

27. T. H. Chrzanowski, R. D. Crotty, J. G. Hubbard and R. P. Welch, Microbial ecology, 1984, 10, 179-185.

28. P. Breeuwer, J. L. Drocourt, N. Bunschoten, M. H. Zwietering, F. M. Rombouts and T. Abee, Appl. and Environm. Microbiology, 1995, 61, 1614-1619.

29. W. Ganley, P. Ryan, J. Van Duijneveldt, J. Colloid Interf. Sci., 2017, 505, 139-147.

\section{ORCID}

Anupam A. K. Das: $\quad$ 0000-0003-1948-8811

Vesselin N. Paunov: 0000-0001-6878-1681 